\title{
Experimental study of the spatial distribution of quantum correlations in a confocal Optical Parametric Oscillator
}

\author{
M. Martinelli, N. Treps, S. Ducci, S. Gigan, A. Maître, C. Fabre \\ Laboratoire Kastler Brossel, Université Pierre et Marie Curie, \\ case 74, 75252 Paris cedex 05, France.
}

(Dated: November 4, 2018)

\begin{abstract}
We study experimentally the spatial distribution of quantum noise in the twin beams produced by a type II Optical Parametric Oscillator operating in a confocal cavity above threshold. The measured intensity correlations are at the same time below the standard quantum limit and not uniformly distributed inside the beams. We show that this feature is an unambiguous evidence for the multimode and nonclassical character of the quantum state generated by the device.
\end{abstract}

PACS numbers: 42.50.Dv; 42.65.Yj; 42.30.-d; 42.50.Lc 


\section{INTRODUCTION}

To date, almost all the experiments investigating the quantum properties of the states of light produced by Optical Parametric Oscillators (OPO) have been performed through the measurement of the total intensity of the generated fields, obtained by integrating on the detector the whole wavefront intensity distribution. Such measurements have put in evidence the squeezed vacuum character of the output of a degenerate OPO below threshold [1], the quantum intensity correlations between the signal and idler beams (twin beams) [2], and the bright squeezing of the output of an optical parametric amplifier (OPA) [3] and of the pump beam reflected by an OPO [4].

In the last years, the interest has turned to the spatial aspects of quantum fluctuations, in particular because they open new perspectives in the quantum information field : spatial features offer the possibility of parallel processing and multichannel operation, each part in a transverse section of a beam playing the role of a channel. The concepts of temporal squeezing and correlations of light beams as a whole have been extended to spatio-temporal properties for the local quantum fluctuations in the plane perpendicular to the propagation : squeezing of the temporal fluctuations inside a small part of the transverse plane, or temporal quantum correlations between different transverse areas of the beam are some of the manifestations of the transverse quantum properties of light. It has been shown that, in order to obtain strong spatial quantum effects, one needs to use multimode nonclassical states of light, in contrast to the single mode operation of the experiments quoted above. It has also been shown that such nonclassical multimode states can be used to improve the optical resolution [5] and to measure small transverse displacements of a light beam below the standard quantum limit [6, 7].

Our investigations of the spatial distribution of multimode states of light produced by OPOs are related to the pioneer studies of the stochastic spatial noise performed in gas lasers [8], and more recently in semiconductor lasers (diode lasers and VCSELs) [9], for which the spatial distribution of the quantum fluctuations of the output beam has been studied and interpreted in terms of a superposition of several Hermite-Gauss modes. They must also be connected to the studies of spatial quantum effects for the photons generated by spontaneous parametric down-conversion, at the photon counting level, for example to the recent demonstration of spatial antibunching [10].

Parametric interaction in a non-linear crystal has been theoretically shown to be a very efficient source of multimode nonclassical states of light. Many theoretical studies [11, 12] have shown in particular that Optical Parametric Amplifiers (OPA) generate multimode squeezed states. OPOs 
have also been shown to be a source of multimode squeezed states provided that they use optical cavities with degenerate transverse modes : in particular, detailed theoretical investigations have been performed on sub-threshold OPOs in planar [13, 14], or quasi-planar cavities [15, 16].

From an experimental point of view, such cavities, which are close to instability, are quite difficult to handle and have very high oscillation thresholds. Confocal cavities are much easier to operate, and still exhibit interesting nonclassical transverse effects, such as multimode squeezing in the degenerate case below threshold [17]. The multimode transverse operation of confocal OPOs above threshold has been theoretically investigated at the classical level [18], and experimentally put in evidence in [19], where it has been shown that the field emitted by a confocal OPO above threshold can be described as a superposition of a great number of $T E M_{p q}$ modes. The quantum description of this regime is unfortunately a difficult task, as the device switches from an uncoupled regime of the different transverse modes to a strongly coupled regime when passing from the sub-threshold emission to the regime of intense output.

This paper is devoted to the study of the spatial distribution of quantum fluctuations of such a device, and shows that it generates multimode nonclassical states of light. In section II, we give the precise definition of multimode quantum states of light. We present in section III a criterion unambiguously characterizing such states in our experimental configuration, namely the measurement of the spatial distribution of the intensity correlations between the signal and idler beams. Section IV presents the experimental setup and the procedure for measuring these correlations. Finally, section $\mathrm{V}$ presents the experimental results proving that the signal and idler beams emitted by the confocal OPO are multimode and spatially quantum correlated.

\section{INTRINSIC CHARACTERIZATION OF MULTIMODE AND SINGLE MODE BEAMS}

\section{A. Definition of single mode and multimode beams}

In the studies on optical patterns, a beam is said to be multimode (in the $T E M_{p q}$ basis), when the far field patterns appear to be different from the near field patterns. Actually, this feature is a proof that the beam consists of a superposition of several $T E M_{p q}$ modes. The electric field positive frequency envelope of such a light beam, $E^{(+)}(\vec{r}, z)$, expanded in the basis of $T E M_{p q}$ modes $u_{p, q}(\vec{r}, z)$, writes :

$$
E^{(+)}(\vec{r}, z)=\sum_{p, q} \alpha_{p, q} u_{p, q}(\vec{r}, z) .
$$


with more than one $\alpha_{p, q}$ coefficient different from zero. Nevertheless, if the $\alpha_{p, q}$ coefficients are fixed (i.e. if we deal with a coherent superposition of modes and not a statistical one), one can always define a new mode $v_{0}(\vec{r}, z)$ :

$$
v_{0}(\vec{r}, z)=\frac{E^{(+)}(\vec{r}, z)}{\int\left|E^{(+)}(\vec{r}, z)\right|^{2} d^{2} r}
$$

and construct a new orthonormal basis of modes $\left\{v_{i}(\vec{r}, z)\right\}$ in which $v_{0}$ is the first element. In this new basis, the field which appeared multimode in the Gauss-Laguerre basis, is proportional to $v_{0}$ and is then single mode. This simple reasoning at the classical level seems to show that the single or multimode character of a beam having a well defined and fixed amplitude distribution depends on the choice of the basis, and is not an intrinsic property. A $T E M_{00}$ laser beam is single mode in the Laguerre-Gauss basis, and multimode in the basis of transverse plane waves. We will show here that this statement is no longer true when one describes the beam at the quantum level.

We will now define a single mode quantum state of light in the following way : $\mid \Psi>$ is a single mode quantum state of light if there exists a basis of modes $\left\{v_{i}(\vec{r}, z)\right\}$ in which it can be written as

$$
|\Psi>=| \Psi_{0}>\otimes|0>\otimes| 0>\otimes \ldots
$$

where the first transverse mode $v_{0}$, whatever its shape, is a non-vacuum state $\mid \Psi_{0}>$, and all the other modes are in the vacuum state. We will call intrinsic multimode states all states defined by a ket $\mid \Psi>$ which cannot be written as (3) in any basis. We will give in the next subsection a characterization of such single mode or multimode states which is independent of the basis used to describe it.

This striking difference between the classical and quantum description of a multimode state comes from the fact that a quantum state gives information on the mean electric field, but also on the statistical distribution of quantum fluctuations : a single mode beam is characterized by a well defined transverse variation which carries all its transverse information. Consequently, in such a state the transverse distribution of quantum fluctuations can be deduced from the transverse variation of the mode itself. In contrast, in intrinsic multimode states, the spatial distribution of fluctuations and correlations cannot be deduced from the structure of the mean field.

Let us finally mention that this problem is modified if one considers stochastic fields, i.e. having classical fluctuations. In this case also, the comparison between the spatial (or temporal) distribution of the mean field and of the classical fluctuations is still an intrinsic tool to determine whether the field can be considered as single mode or multimode [9]. We will not consider further this problem here. 


\section{B. Characterization of a single mode quantum state}

Let us consider a single mode state of light $|\Psi\rangle$, written in the adapted basis $\left\{v_{i}\right\}$ as (3), and let us call $\hat{a}_{i}$ the annihilation operator of photons in the mode $v_{i}$. Let us now introduce any other

mode basis of the transverse plane $\left\{w_{j}\right\}$, and the corresponding annihilation operators $\hat{b}_{j}$. There is a unitary transformation relating the two basis, and correspondingly the two sets of annihilation operators :

$$
\hat{b}_{i}=\sum_{j} U_{i j} \hat{a}_{j}
$$

From equations (3) and (41), one deduces that:

$$
\hat{b}_{i}\left|\Psi>=U_{i 0} \hat{a}_{0}\right| \Psi_{0}>
$$

We thus obtain a specific property of a single mode quantum state : the action on it of all the annihilation operators of any given basis of transverse modes gives vectors which are all proportional. One can show that this feature is a necessary and sufficient condition to be a single mode state [20]. In contrast, for an intrinsic multimode state, there exists at least one basis of modes in which this property is not true. The condition for single mode states being very restrictive, the set of single mode states is a very small subset of the general Hilbert space. The complementary of this subspace, namely the set of intrinsic multimode states, is therefore much larger.

This characterization of single and multimode states appears to be quite mathematical, and seems difficult to implement experimentally. We give in the following a more convenient property of single mode states, which is not a necessary and sufficient condition, but which can be submitted to an experimental check with our set-up.

\section{CHARACTERIZATION OF SINGLE MODE AND MULTIMODE TWIN BEAMS}

\section{A. Partial measurement of intensity fluctuations on a single mode beam}

Let us consider an intensity, or photon number, measurement using a detector of variable transverse area $S_{A}$. We have shown in reference [6] that if the light is in a single mode state, the variance of the photon number fluctuations measured with this partial detector is given by :

$$
\Delta N_{A}^{2}=<N_{A}>+\frac{<N_{A}>^{2}}{<N_{t o t}>^{2}}\left(\Delta N_{t o t}^{2}-<N_{t o t}>\right)
$$


where $\left\langle N_{A}>\right.$ is the mean number of photons detected on the area $S_{A},<N_{t o t}>$ the mean number of photons detected on the whole transverse plane, and $\Delta N_{\text {tot }}$ the corresponding variance. One sees that the intensity noise normalized to shot noise, $\frac{\Delta N_{A}^{2}}{\left\langle N_{A}\right\rangle}$ varies linearly with the quantity $T=\frac{\left\langle N_{A}\right\rangle}{\left\langle N_{t o t}\right\rangle}$. This formula can be understood by considering that in a single mode state the photons are randomly distributed in the transverse plane, so that a partial detection will introduce sorting noise in the detection of the photon number, exactly like when one introduces a linear loss of value $T$ in front of the detector. This intuitive picture is no longer true for multimode beams.

\section{B. Partial measurement of intensity correlations on single mode twin beams}

In a non degenerate OPO, the emitted signal and idler fields present strong intensity correlations at

the quantum level ("twin beams"). In particular, the variance of the difference $N_{d i f, t o t}=N_{1, t o t}-N_{2, t o t}$ between the signal (labeled 1) and idler (labeled 2) intensities detected over the whole transverse plane is smaller than $\left\langle N_{1, t o t}\right\rangle+\left\langle N_{2, t o t}\right\rangle$, which is the shot noise for the sum of the signal and idler beams. When the beams produced by the OPO are both single mode, one can easily show from [6] that the following formula, similar to (6), holds in case of a partial photodetection of signal and idler beams by two detectors having the same areas and positions $S_{A}$ in the two beams :

$\Delta N_{d i f, A}^{2}=\left(<N_{1, A}>+<N_{2, A}>\right)+\frac{\left(<N_{1, A}>+<N_{2, A}>\right)^{2}}{\left(<N_{1, t o t}>+<N_{2, t o t}>\right)^{2}}\left(\Delta N_{d i f, t o t}^{2}-\left(<N_{1, t o t}>+<N_{2, t o t}>\right)\right)$

Similarly to the interpretation of formula (6), this formula can be understood by considering that in single mode twin beams there is no spatial correlations between the twin photons inside the signal and idler beams, the photons being randomly distributed inside the two beams. If, instead of small detectors, we use detectors with a broad enough area to detect the whole beams, but preceded by a diaphragm, or iris, of variable transmission $T$, expression (7) shows that in single mode twin beams the relative noise on the intensity difference, $n_{A}=\frac{\Delta N_{d i f, A}^{2}}{\left\langle N_{1, A}\right\rangle+\left\langle N_{2, A}\right\rangle}$, is a linear function of the transmitted mean intensity. In contrast, a nonlinear variation of this quantity will be a signature for intrinsic multimode twin beams, for which the twin photons are not randomly distributed inside the two beams. 


\section{Partial measurement of intensity correlations on single mode twin beams undergoing} different losses

We have assumed so far that the detected mean intensities of the output beams are always equal, even in partial photodetection. In real experiments, this is not exactly the case, and imbalances of several percents are commonly measured, especially in confocal OPOs, where the patterns observed in the signal and idler beams are different. The imbalance will affect the intensity fluctuations of the two beams in a different way. The imbalance of the whole beams is due to the fact that the signal and idler beams, produced in exact equal amounts in the nonlinear crystal, experience different losses in their propagation. Furthermore, in the case of a partial photodetection, one must also take into account that the signal and idler beams may have different shapes. It is important to precise the way to define the "twin character" of such imbalanced beams in a partial measurement. We will first introduce the normalized intensity difference noise $n$ when the whole beams are measured (Fig. 11a). We will then consider a partial measurement (Fig.1]b) and define the direct normalized intensity difference noise $n_{d}$, with no corrections, as it can be directly calculated from the measurement. We will finally define the corrected normalized intensity difference noise $n_{\text {corr }}$, an attempt to recover the value of $n$ from $n_{d}$ assuming that the beams are single mode.

Assuming that the mean signal and idler fields are real, we can write the field intensity operator, in the small fluctuation approximation, as :

$$
\delta \hat{i}_{m}=2 E_{m} \delta \hat{E}_{m} \quad m \in\{1,2\}
$$

where $\delta \hat{E}_{m}$ is the quadrature fluctuation operator in the amplitude direction. We now introduce the normalized quantity $n$, which is the variance of the intensity difference fluctuations normalized to the total shot noise, and can be written as :

$$
n=\frac{\left\langle\left(\delta \hat{i}_{1}-\delta \hat{i}_{2}\right)^{2}\right\rangle}{4\left(i_{1}+i_{2}\right) \Delta^{2} E_{v}}
$$

where $\Delta^{2} E_{v}$ is the noise variance of the vacuum field. $n>1$ for beams with classical correlations (obtained for example by using beamsplitters) and $n<1$ for quantum correlated beams ("twin beams"). Let us now assume that the two beams are subject to losses before being incident on the photodetectors (Fig.1 $1 \mathrm{~b})$. We call $E_{m}^{\text {out }}$ the fields which are incident on the photodetectors and $i_{m}^{\text {out }}$ their intensities, and $n_{d}$ the normalized variance of their intensity difference :

$$
n_{d}=\frac{\Delta^{2} i_{d}^{\text {out }}}{4\left(i_{1}^{\text {out }}+i_{2}^{\text {out }}\right) \Delta^{2} E_{v}}=\frac{\left\langle\left(\hat{\hat{i}_{1}^{\text {out }}}-\hat{\delta \hat{i}_{2}^{\text {out }}}\right)^{2}\right\rangle}{4\left(i_{1}^{\text {out }}+i_{2}^{\text {out }}\right) \Delta^{2} E_{v}}
$$



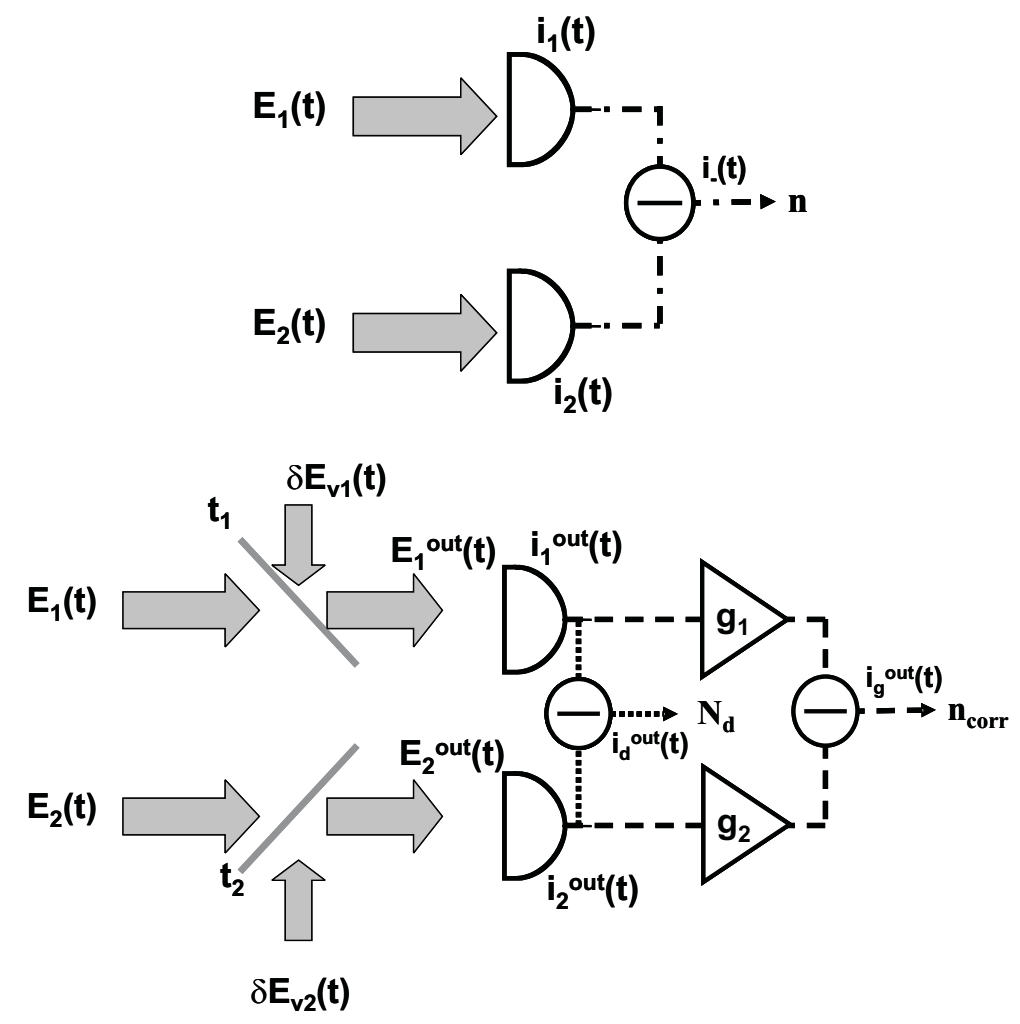

FIG. 1: measurement of twin beams correlation in the imbalanced case. $i_{d}^{\text {out }}$ : direct difference measurement. $i_{g}^{\text {out }}$ : difference measurement after gain corrections.

In the transverse single mode case, losses of any origin and partial photodetection have the same effect on the fluctuations mechanism, identical to the effect of a beam splitter of transmission $t_{m}(\mathrm{~m}=1,2)$ (with $t_{m}^{2}=i_{m}^{\text {out }} / i_{m}$ ). The field intensity $\hat{i}_{m}^{\text {out }}$ measured by the photodetectors present fluctuations $\delta \hat{i}_{m}^{\text {out }}(\mathrm{t})$ given by:

$$
\delta \hat{i}_{m}^{\text {out }}(t)=2 t_{n}^{2} E_{m} \delta \hat{E}_{m}+2 t_{m} \sqrt{\left(1-t_{m}^{2}\right)} E_{m} \delta \hat{E}_{v m} \quad m \in\{1,2\}
$$

Knowing the effective loss coefficient $t_{m}$, it is possible to adjust the gain $g_{m}$ on the acquisition channels at the value $1 / t_{m}^{2}$, as shown in fig. 1, so that the mean values of the corrected intensities $g_{m}\left\langle\hat{i}_{m}^{\text {out }}\right\rangle$ are equal to their value without losses $\left\langle\hat{i}_{m}\right\rangle$. If one assumes that the two beams are single transverse mode, it is possible to recover the original normalized intensity difference noise $n$ of eq. (9) by subtracting the effect of the vacuum fluctuations $\delta \hat{E}_{v m}$. Writing $\Delta^{2} i_{g}^{\text {out }}$ the fluctuations of the difference between the two acquisition channels :

$$
\Delta^{2} i_{g}^{\text {out }}=\left\langle\left(g_{1} \delta \hat{i}_{1}^{\text {out }}-g_{2} \delta \hat{i}_{2}^{\text {out }}\right)^{2}\right\rangle
$$


One easily shows that the quantity

$$
n_{\text {corr }}=\frac{\Delta^{2} i_{g}^{\text {out }}}{4\left(g_{1} i_{1}^{\text {out }}+g_{2} i_{2}^{\text {out }}\right) \Delta^{2} E_{v}}+1-\frac{g_{1}^{2} i_{1}^{\text {out }}+g_{2}^{2} i_{2}^{\text {out }}}{g_{1} i_{1}^{\text {out }}+g_{2} i_{2}^{\text {out }}}
$$

is equal to $n$ in the single transverse mode case. The two last terms of (13) correspond to the corrections arising from vacuum fluctuations introduced by the losses or the partial detection.

\section{Multimode twin beams}

Since for single mode signal and idler beams $n_{\text {corr }}=n$, a constant value $n_{\text {corr }}$ with the transmittance $t_{m}$ for a partial measurement (or a linear variation of $n_{d}$ ) is a good indication (but not a proof) of the single mode character of the signal and idler mode beams. In contrast a non constant value of $n_{\text {corr }}$, or a nonlinear variation of $n_{d}$, with respect to the losses is a an unambiguous signature of the multimode character of the two beams generated by the OPO. Furthermore, if these quantities are smaller than 1, we can conclude that we are in presence of multimode nonclassical beams. Strictly speaking, as the vacuum correction in $n_{\text {corr }}$ has been derived assuming single mode beams, $n_{\text {corr }}$ do

not anymore correspond exactly to a noise correlation in the case of multimode beams. Moreover, for a partial measurement, the mean intensity of both beams may be different, and neither $n_{d}$ nor $n_{\text {corr }}$ are perfectly suited for the exact characterization of quantum intensity correlation. For these reasons, in the case of a partial measurement of a multimode beam, more sophisticated criteria should be investigated, that will be developed in a forthcoming publication.

\section{EXPERIMENTAL SETUP}

For OPOs operating in cavities with degenerate transverse modes, the formation of complex spatial structures has been theoretically [21, 22] and experimentally [19, 23] studied. Furthermore, quantum multimode operation of OPO has been already theoretically predicted [14]. But, up to now, to our knowledge, there has been no experimental demonstration that the emission of an OPO is intrinsically multimode. The experiment presented here investigates the spatial distribution of the intensity correlation between the signal and idler beams emitted by a confocal OPO above threshold in order to identify their single mode or multimode character. The setup, shown in Fig.2, can be divided in two parts: the triply resonant OPO and the acquisition system of the intensity fluctuations of the beams. 


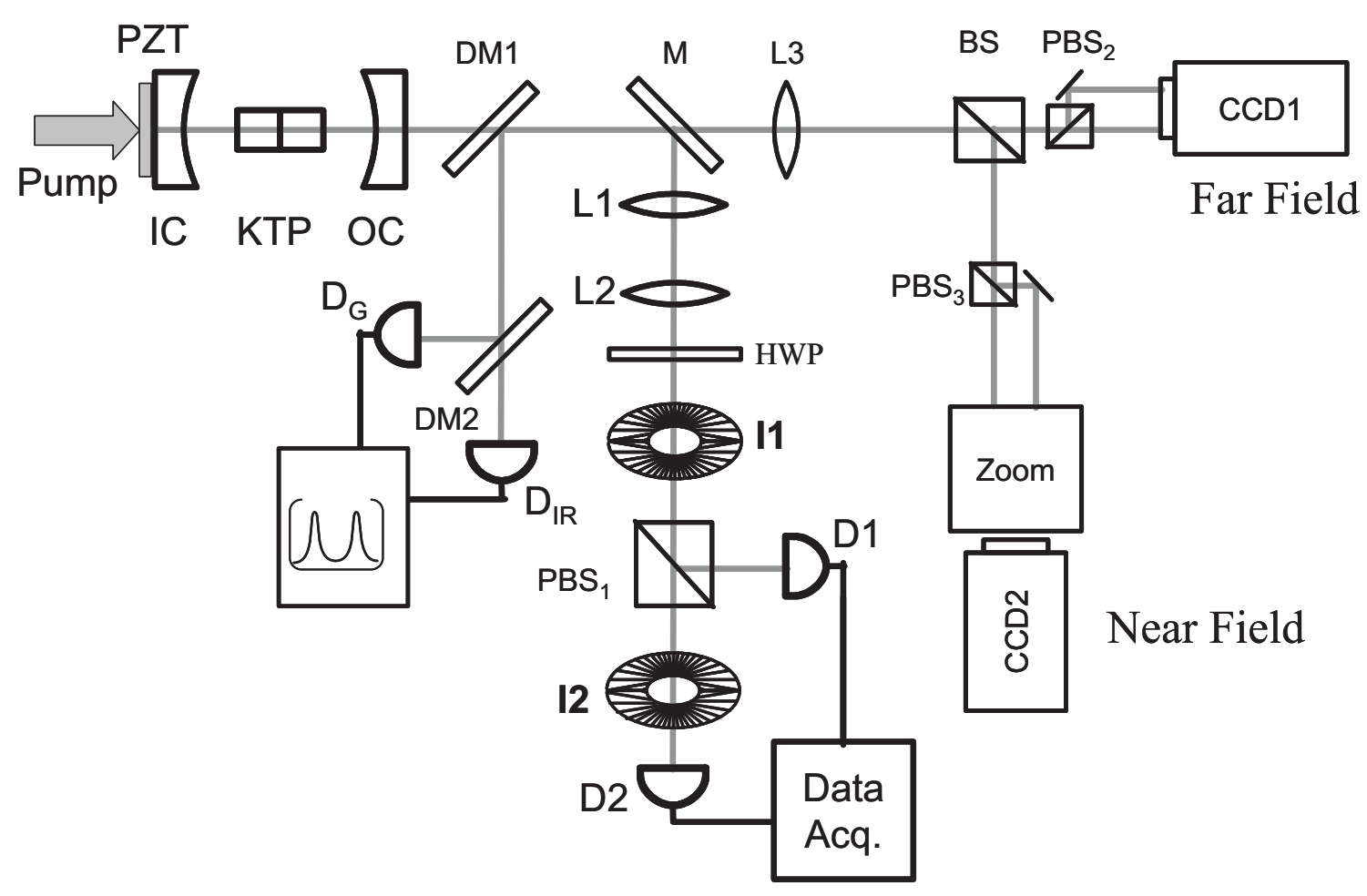

FIG. 2: Experimental setup

\section{A. The triply resonant OPO}

In order to match the desired requirements of power and stability of the experiment, a dedicated setup was made for the generation of the pump beam for the OPO. We used an ultrastable single mode Nd:YAG (yttrium aluminum garnet) laser with $350 \mathrm{~mW}$ of output power to lock a flash-lamppumped Nd:YAG laser operating in a ring cavity. This configuration [19, 23 gives a stable single mode beam with an output power of $3.5 \mathrm{~W}$ at $1064 \mathrm{~nm}$. This beam is injected in a semi-monolithic frequency doubler, using a $\mathrm{MgO}: \mathrm{LiNbO}_{3}$ crystal and a concave mirror. The output of the second harmonic generator reproduces the frequency stability of the injected Nd:YAG laser and gives a total output power of $1.3 \mathrm{~W}$ at $532 \mathrm{~nm}$. The pump is then injected inside the OPO with a waist equal to twice the $T E M_{00}$ waist of the OPO cavity mode like in reference [19].

The nonlinear crystal used inside the OPO is a walk-off compensated KTP (potassium titanyl phosphate) cut for type II phase matching. It is made by two $5 \mathrm{~mm}$ long crystals that are optically contacted, with their orientations compensating the walk-off effect. The triply resonant OPO uses two plane-concave mirrors, whose curvature is $\mathrm{R}=100 \mathrm{~mm}$. The input coupler (IC) has a transmittance of $10 \%$ for the pump (532 nm) and high reflectance for the Nd:YAG wavelength (1064 nm). The 
output coupler (OC) has a high reflectance at $532 \mathrm{~nm}$ and 1\% transmittance at $1064 \mathrm{~nm}$. Measured finesse value of the cavity with the crystal is 40 at $532 \mathrm{~nm}$ and 300 at $1064 \mathrm{~nm}$ and temperature is controlled such that signal (o-polarization) and idler (e-polarization) emitted by the OPO are close to frequency degeneracy. The cavity length can be tuned over a few free spectral ranges with a piezo-electric ceramic (PZT) attached to the input coupler. A coarse control of the length of the OPO is performed by means of translation stages fixed on both cavity mirrors. A fraction of the infrared output of the Nd:YAG laser can also be injected into the cavity for alignment purposes and to check the transverse mode separation. The OPO works close to the confocal configuration. In order to define the region of confocality, it is useful to remember that the distance L between two spherical mirrors for a confocal cavity depends not only on the ray of the concave surface but also on the refractive index of the medium inside the cavity. Therefore, considering the diffraction effects inside a crystal of length $\ell$ and refractive index $n$ in a cavity made by two spherical mirrors of ray $R$, the distance $\mathrm{L}$ between the mirrors for the confocality will be [24]

$$
L_{\text {conf }}=R+\ell(1-1 / n)
$$

Since the refractive index for pump, signal and idler are different (respectively 1.7881, 1.8296 and 1.7467 [25]), the length of the cavity $L_{\text {conf }}$ for which the exact confocality is obtained, is different for each of the three modes $(104.41,104.28$ and $104.53 \mathrm{~mm})$. Transverse degeneracy is achieved when different transverse modes are resonant for the same cavity length. Due to the width of the resonance peaks, transverse degeneracy can be obtained even if the cavity is not exactly confocal. The range of confocality can be defined as the region of the cavity length where the fundamental and the first transverse even mode separation is smaller than the cavity bandwidth, and, following [26], can be expressed as

$$
\left|L-L_{\text {conf }}\right|<\frac{\pi R}{2 F}
$$

where $\mathrm{F}$ is the finesse of the cavity. In our case, the range of confocality for signal, idler (and pump) is larger than the difference between the confocal lengths for the signal, idler and pump modes. Transverse degeneracy, essential for multimode emission, is then achievable at the same time for the three modes. Taking the average of the confocal length for signal and idler, we will consider the confocal length as $L_{\text {conf }}=104.41 \mathrm{~mm}$, and express the cavity length in terms of $\Delta L=L-L_{\text {conf }}$. Although the threshold for oscillation is quite low and around $30 \mathrm{~mW}$, significant thermal effects take place inside the cavity even close to threshold, because of the non-negligible absorption of the green light $(3 \%$ at $532 \mathrm{~nm})$ and of infrared light $(0,45 \%$ at $1064 \mathrm{~nm})$ : thermal lensing changes the total 
Gouy phase shift added to the wave in a round trip [26] and even a sub-confocal cavity can become transverse degenerate if the pump power injected into it is sufficiently high. Therefore the confocality [19] is obtained for cavity lengths which are shorter than the confocal length $L_{\text {conf }}$, defined above for a "cold" OPO with no thermal effects.

\section{B. Detection and acquisition}

The details of the setup used for studying the transverse distribution of noise in twin beams cavity can be seen in Fig.2. Near the output coupler, a dichroic mirror (DM1) is used to eliminate the residual green light transmitted through the OPO cavity. A small fraction $(2 \%)$ of the infrared light that is reflected by this mirror and transmitted by a second dichroic mirror (DM2) is monitored by an InGaAs infrared photodetector $D_{I R}$. It is used to stabilize the OPO cavity by a servo-loop made by a high-voltage amplifier connected to the PZT, controlling the output power of the IR beam and stabilizing it during a time ranging from seconds to minutes of continuous operation. The green light reflected by DM1 and DM2 is detected by a visible photodetector $D_{G}$.

After the dichroic mirror (DM1) at the OPO output, a flipping mirror (M) allows either the imaging of signal and idler far and near fields on a screen, or the recording of their intensities by two InGaAs infrared four-quadrants photodetectors D1 and D2 (ETX505Q from Epitaxx). In this experiment, only one quadrant of each photodetector is used. Their quantum efficiency are very close (less than $1 \%$ difference) and equal to $90 \% \pm 5 \%$. In both configurations, polarizing beamsplitters (PBS)

separate the signal and idler beams. Depending of the orientation of the half wave plate (HWP) placed before PBS1, signal and idler can be sent either to D1 or D2. During the measurement process, two irises of variable diameter are used to select in the far field a circular region of the output beam. Iris I1 is used to select a narrow circular region on both signal and idler, while iris I2 acts only on a single beam, either signal or idler. The lenses L1 and L2 are adjusted for each experiment in order to project the far field of the beam into the iris plane. The transmitted intensities are recorded and their fluctuations are monitored by a dedicated data acquisition system that is described below.

The data acquisition system used for the noise measurement differs from the usual method of direct measurement and subtraction of noise fluctuations (e.g. Ref.[27). The high frequency part of the photocurrent of each photodetector is amplified by a transimpedance amplifier and a broadband 36 $\mathrm{dB}$ amplifier. This signal is then mixed in an electronic demodulator at a frequency $f_{0}$ equal to 3.5 MHz which lies inside the cavity bandwidth for the infrared modes and above the excess noise 
frequencies of the output beams. The output of the mixer has an active low-pass filter, working at $100 \mathrm{kHz}$. This signal is registered by a fast Analog-to-Digital acquisition card for measuring the noise correlation of the photocurrents. We used two acquisition cards (PCI6110E from National Instruments) with four simultaneous measurement channels each and 12 bits for signal conversion. The signal is measured with a repetition rate of $200 \mathrm{kHz}$ and registered in the computer. Remaining channels of the data acquisition system are used for the measurement of the average value of the photocurrent of each detector, as well as the photocurrents of detectors $D_{G}$ and $D_{I R}$. From the stored information of the average value and noise fluctuations of the photocurrent we can calculate the noise correlation of the intensity, and compare it to a previous calibration of the shot noise level made with a single output of the OPO or with the injected IR light from the Nd:YAG laser. This technique allows us to acquire in a very short time interval both the temporal fluctuations of a given Fourier component of the photocurrents and of their mean values, and then to postprocess the stored data. We can thus determine the different normalized quantities that we have defined earlier in that paper.

\section{Experimental procedure}

When the OPO oscillation is stabilized for a given cavity length, we perform two kinds of experiments. In the first experiment, we record the simultaneous noise distribution of the signal and idler beams by using the iris I1. We continuously close the iris I1 in $2 \mathrm{~s}$, while acquiring a long series of values of $\delta i_{s}, \delta i_{i}, i_{s}, i_{i}, i_{I R}$ and $i_{G}$ (6 series of 400,000 simultaneous values). We analyze the data by groups of 10,000 values, calculating for each group the average photocurrent value $\left\langle i_{m}\right\rangle(\mathrm{m}=1,2)$ and the normalized variances $n_{\text {corr }}$ and $n_{d}$. The corresponding vacuum fluctuation $\Delta^{2} E_{v}$ was previously calibrated, and the electronic noise level is subtracted in the calculation of the variances.

The transmittance of the iris for each series of 10000 points is defined as $T=\frac{r}{r_{\text {open }}}$ where $r$ is the ratio $r=\frac{\left\langle i_{s}+i_{i}\right\rangle}{\left\langle i_{I R}\right\rangle}$, and $r_{\text {open }}$ corresponds to the initial value of $r$ when the diaphragm is open. The normalized intensity difference noise variances $n_{\text {corr }}$ and $n_{d}$ are then plotted as a function of the transmission of the iris.

The second kind of experiments consists in setting iris $I_{2}$ on either the signal or the idler beam path and taking the unaffected beam as a reference. The procedure is identical to the previous one, except that $r$, for the iris on the signal beam, is defined as $r=\frac{\left\langle i_{s}\right\rangle}{\left\langle i_{I R}\right\rangle}$. Closing the iris $I_{2}$ attenuates only one of the beams, and produces a strong imbalance between the photocurrents of the two photodetectors, 
with a ratio $\left(i_{s} / i_{i}\right)$ well out of the range $90 \%$ to $110 \%$, which was typical in the previous conditions. With such a large imbalance, the value of $n_{d}$ deduced from the experiment does not give any quantitative information on the quantum correlations between the two beams. On the other hand, the calculation of the corrected value of the normalized noise $n_{\text {corr }}$ allows one to recover the information on the quantum correlation only in the case where the two beams are single mode. Therefore, in this second kind of experiments, we only calculate $n_{\text {corr }}$ as a function of the transmittance $T$.

\section{SPATIAL DISTRIBUTION OF THE INTENSITY CORRELATION}

\section{A. Experiments with the iris on both signal and idler beams}

As described in the previous subsection, only iris $I_{1}$ is present. We have realized in this configuration two series of measurements: in the first one, the OPO oscillation is stabilized for a cavity length outside the confocality range, ensuring a $T E M_{00}$ output mode for signal and idler beams (ref. [19, 23]) ; in the second one, the cavity length is set to a shorter value, inside the confocality range, where complicated patterns can be observed in the signal and idler beams. While closing continuously the

iris $I_{1}$, we record the transmitted intensity of the signal and idler modes and the normalized intensity noise difference variance $n_{d}$. The different normalized variances are then plotted as a function of the transmittance $T$. In the figures (3), the straight line represents the value of $n_{d}$ that could be calculated with a single mode beam having the same intensity correlation as the whole beam. One observes that outside the confocality range, where the signal and idler fields are close to $T E M_{00}$, the calculated straight line fits well the experimental points. Moreover, for an open diaphragm, the normalized intensity difference noise variance is equal to 0.8 , demonstrating significant quantum intensity correlations between signal and idler.

In the confocality range, where the signal and idler fields have more complicated, and different, transverse variations, one observes that the variation of $n_{d}$ is no longer linear with the transmittance, demonstrating that the emission of the OPO is multimode. Moreover, when the iris is open in that confocal configuration, the noise correlation is still below shot noise and close to the value obtained for the single mode emission: the quantum intensity correlations for the whole beams are preserved even for multimode beams. The increase of noise when the iris is closed shows that the intensity correlation is stronger in the outer parts of the beams than in the center. Unfortunately, with such an experiment it is difficult to describe more precisely the transverse distribution of correlations 

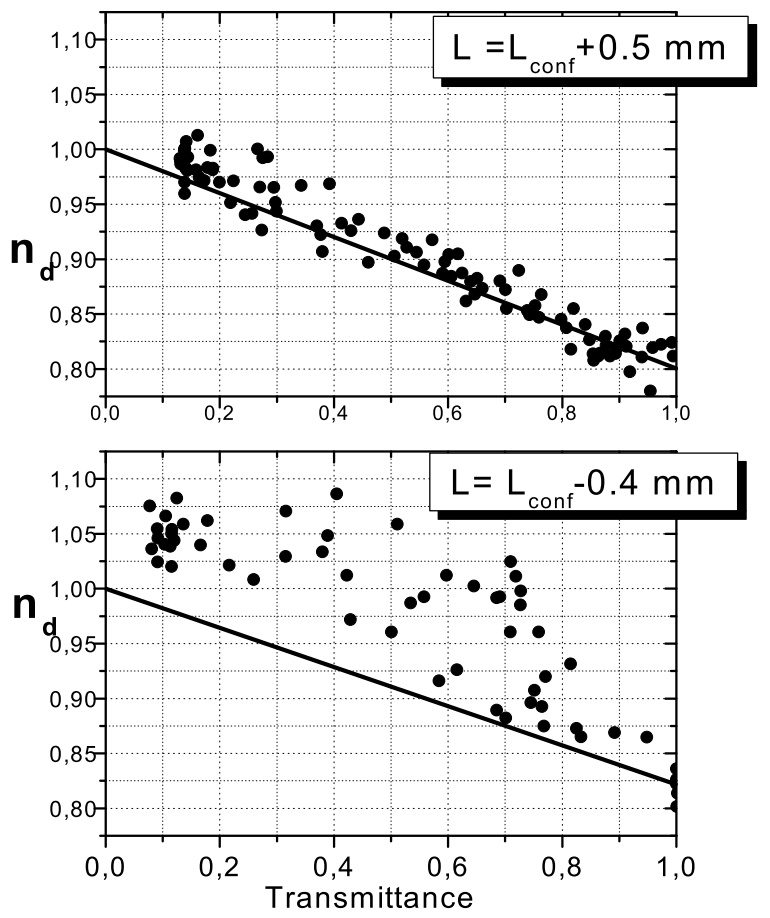

FIG. 3: spatial distribution of the normalized intensity difference noise $n_{d}$ as a function of the transmittance T with iris $I_{1}$ on signal and idler beams. Points: values of $n_{d}$ for a cavity length equal to $L_{\text {conf }}=L+0,5 \mathrm{~mm}$ (beyond the confocality range)and $L_{c o n f}=L-0,4 m m$ (inside the confocality range). Straight line: Values of $n_{d}$ that would be obtained with a single mode beam having the same squeezing when the iris is fully open.

between the signal and idler beams.

\section{B. Experiments with the iris either on signal or idler beam}

\section{Single mode beams}

For a length of cavity $L=L_{\text {conf }}+5,6 \mathrm{~mm}$, where the OPO is supposed far from transverse degeneracy, we compared the results of the two kinds of experiments for the values of $n_{\text {corr }}$ as a function of the transmittance. The results are plotted in Fig. 1. For all those experiments, as the beam intensity on the photodetector is reduced, the fluctuations of the photocurrent approaches the electronic level. The dispersion in the calculated data increases when we close the iris. Typically, the variance of the intensity noise reached the dark noise level for an incident power of $0.2 \mathrm{~mW}$, obtained 

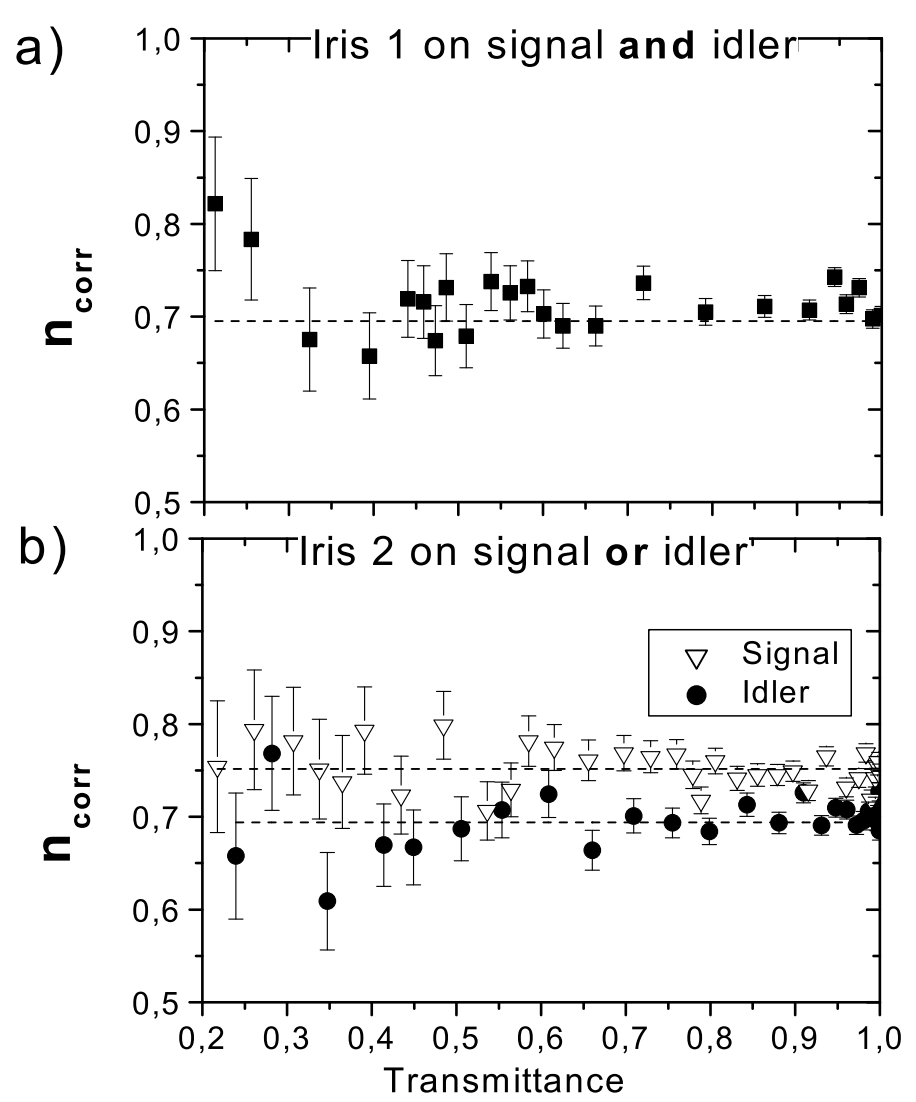

FIG. 4: Experimental values of $n_{\text {corr }}$ for quasi-TEM $E M_{00}$ signal and idler beams. a) Iris on both beams b)Iris on a single beam. Idler $(\bullet)$ and signal(o). Dashed lines: average corrected noise.

with a typical iris transmittance of $10 \%$.

One observes that all the experimental points are aligned on a horizontal straight line, with a mean value around $70 \%$, which shows that the signal and idler beams are single mode, quantum-correlated, beams. The difference in the obtained level of noise for in fig $4 \mathrm{~b}(69 \%$ and $75 \%)$ is certainly due to the fact that both experiments are performed at different moments, and therefore on possibly different longitudinal modes. The values of $n_{\text {corr }}$ remain stable during a single series of measurements when we close the iris.

\section{Beams emitted by an $O P O$ close to confocality}

The length for the cavity is chosen to be very close to the exact confocal length $L_{\text {conf }}$. In this configuration complex structures appear in far and near fields of the signal and idler beams [19]. We 
performed the same experiments and analysis as in the previous subsection, adding the image of the near field and the far field of the beam obtained with the CCD cameras. Fig. 5a, b and c display respectively the results obtained for $L-L_{\text {conf }}=0.38,-0.37$ and $-0.62 \mathrm{~mm}$. In the configuration of

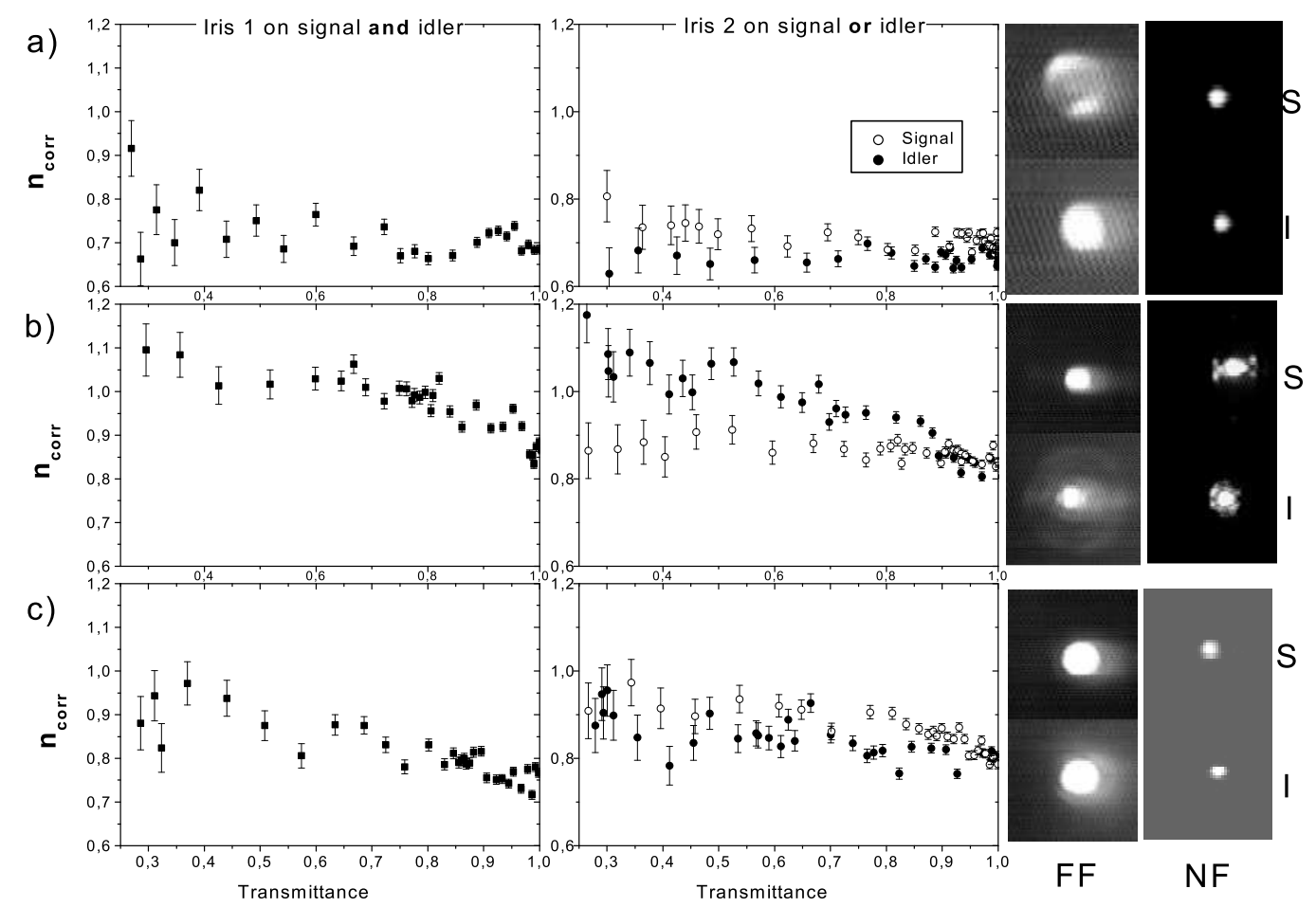

FIG. 5: Experimental values of $n_{\text {corr }}$ for signal and idler beams displaying complex transverse distributions. The distance from confocality $(\Delta L)$ is equal respectively to a) $0.38, \mathrm{~b})-0.37$, and c) $-0.62 \mathrm{~mm}$. Left, iris on both beams: $(\boldsymbol{\square})$. Center, iris on a single beam. Idler $(\bullet)$ and signal(o). Right: Far field (FF) and near field (NF) images of signal and idler beams.

Fig.5a, one observes a nearly gaussian distribution of the idler beam and a non-gaussian distribution of the signal beam. When the iris $I_{2}$ is placed on the idler beam, $n_{\text {corr }}$ does not deviate in a clear way from a flat line, except for lower values of the iris transmittance. In this situation, the dispersion in the results increases, following the attenuation of the idler field intensity. When we set the iris on the signal path, the corrected noise deviates slightly from a constant value for values of the transmittance close to 50\%. The same behavior can be observed when we perform the experiment with iris 1 on both signal and idler.

In the configuration of Fig.5b, more complex structures appear : a ring pattern can be seen in the 
outer part of the idler far field image. In the near field, complex structures appear for both signal and idler beams. In this situation, when iris $I_{1}$ is put on both beams, the corrected correlation noise $n_{\text {corr }}$ goes up to the shot noise level and stabilizes around this value. It is interesting to observe that the level of squeezing obtained is reduced, and the normalized noise for open iris starts at $85 \%$. When the iris $I_{2}$ is put on the signal path and closed, $n_{\text {corr }}$ remains constant: from these observations, one can infer that the photons in the signal beams which are correlated to the idler photons are uniformly distributed inside the signal beam. On the contrary, when the diaphragm $I_{2}$ is set on the idler beam, the plot of $n_{\text {corr }}$ deviates from a flat line. In that case the results are very close to those obtained when the iris $I_{1}$ is set on both signal and idler, showing that most of the correlated photons in the idler are concentrated in the external part of the beam. The center part of the idler is constituted essentially of non-correlated photons.

In the configuration of Fig.5c, both signal and idler beams present a faint external ring out of

the central maximum. In that case, the evolution of the noise when closing iris 1 or closing iris 2 for signal or idler are very close. Both signal and idler corrected noises $n_{\text {corr }}$ are reduced when one closes iris I2, showing that unlike the centers of signal and idler beams, their outer parts are quantum correlated. We see that these experiments give interesting indications on the details of transverse distribution of the correlations inside the beams. Unfortunately, the great quantity of modes oscillating simultaneously in a confocal cavity, which makes a theoretical approach of the system very difficult, and the lack of long term stability of the setup, which induces a large dispersion on the experimental results, prevent us from a more quantitative comparison between theory and experiments, which has been tackled in the case of the multimode VCSEL for example [9].

\section{CONCLUSION}

The results presented in this paper show that the intensity correlations in a confocal OPO are at the same time below the standard quantum limit and not uniformly distributed inside the beams, which is a clear evidence that the quantum state generated by such an OPO is a multimode nonclassical state of light. This experiment showed also that even in the multimode case, the intensity fluctuations of the signal and idler beams remain quantum correlated. It would be very interesting to find a theoretical explanation, even qualitative, to the fact that, in our experimental configuration, the central part of the beams seem to be less quantum correlated than the outer parts. We plan to extend this study in the case of an OPO working in a lower degeneracy cavity, where only some few 
modes are allowed to oscillate simultaneously. The small number of oscillating modes can lead to a detailed theoretical description of the system. Another interesting regime is the operation below threshold, where the comparison with theory is somehow simpler, but which requires a spatially resolved homodyne detection. For a more general point, this demonstration of an intrinsic multimode emission of signal and idler beams in the quantum regime of the above threshold OPO, opens interesting prospects for the study and use of transverse quantum effects in bright beams.

\section{Acknowledgments}

Laboratoire Kastler-Brossel, of the Ecole Normale Supérieure and the Université Pierre et Marie Curie, is associated with the Centre National de la Recherche Scientifique.

This work was supported by the European Commission in the frame of the QUANTIM project (IST2000-26019).

M. Martinelli, on leave from the Instituto de Fisica, Universidade de São Paulo, PO Box 66318 CEP, São Paulo-SP Brazil, wishes to thank Coordenação de Aperfeiçoamento de Pessoal de Nível Superior (CAPES-BR) and Fundação de Amparo à Pesquisa do Estado de São Paulo (FAPESP-BR) for funding. Agnès Maître and Sara Ducci are also at the pôle Matériaux et phénomènes quantiques FR CNRS 2437 Université Paris 7 Denis Diderot.

[1] L.A. Wu, H.J. Kimble, J.L. Hall, H. Wu, Phys. Rev. Letters 57, 2520 (1986)

[2] J. Mertz, T. Debuisschert, A. Heidmann, C. Fabre, E. Giacobino, Optics Letters, 16, 1234 (1991)

[3] K. Schneider, R. Bruckmeier, H. Hansen, S. Schiller, J. Mlynek, Optics Letters 21, 1396 (1996)

[4] K. Kasai, Gao Jiangrui, C. Fabre, Europhysics Letters 40, 25 (1997)

[5] M. I. Kolobov, and C. Fabre, Phys. Rev. Lett. 85, 3789-3792 (2000).

[6] C. Fabre, J. B. Fouet, and A. Maître, Opt. Lett. 25, 76-78 (2000).

[7] N. Treps, U. Andersen, B. Buchler, P.K. Lam, A. Maître, H.-A. Bachor and C. Fabre, Phys. Rev. Letters 88, 203601 (2002).

[8] M. D. Levenson, W. H. Richardson, and S. H. Perlmutter, Opt. Letters 14, 779-781 (1989).

[9] J.-P. Hermier,A. Bramati,A. Z. Khoury,E. Giacobino,J.-Ph. Poizat,T. J. Chang, and Ph. Grangier; J. Opt. Soc. Am. B 162140 (1999). A. Bramati,J.-P. Hermier,A. Z. Khoury, E. Giacobino, P. Schnitzer, 
R. Michalzik,K. J. Ebeling,J.-Ph. Poizat,and Ph. Grangier, Opt. Letters 24, 893 (1999). C. L. Garrido Alzar,S. M. de Paula,M. Martinelli,R. J. Horowicz,A. Z. Khoury,G. A. Barbosa, J. Opt. Soc. Am. B 18, 1189 (2001).

[10] W. A. T. Nogueira, S. P. Walborn, S. Pádua, C. H. Monken, Phys. Rev. Lett. 86, 4009 (2001).

[11] M. I. Kolobov, and I. V. Sokolov, Sov. Phys. JETP 69, 1097 (1989).

[12] M. I. Kolobov, Rev. Mod. Phys. 71, 1539-1589 (1999).

[13] A. Gatti, and L. A. Lugiato, Phys. Rev. A 52, 1675 (1995).

[14] L. A. Lugiato, and A. Gatti, Phys. Rev. Lett. 70, 3868 (1993).

[15] L. A. Lugiato, and I. Marzoli, Phys. Rev. A 52, 4886 (1995).

[16] M. Marte, H. Ritsch, K. I. Petsas, A. Gatti, L. A. Lugiato, C. Fabre, and D. Leduc, Optics Express 3, 71-80 (1998).

[17] L. A. Lugiato, and Ph. Grangier, J. Opt. Soc. Am. B 14, 225-231 (1997).

[18] C. Schwob, P. F. Cohadon, C. Fabre, M. A. M. Marte, H. Ritsch, A. Gatti, and L. Lugiato, Appl. Phys B 66, 685-699 (1998).

[19] M. Vaupel, A. Maître, and C. Fabre, Phys. Rev. Lett. 83, 5278-5281 (1999).

[20] N. Treps, Effets quantiques dans les images optiques Doctoral Thesis Universite Paris VI

[21] G. Izús, M. Santagiustina, M. San Miguel, P. Colet, J. Opt. Soc. Am. B 16,1592 (1999); M. Santagiustina, P. Colet, M. San Miguel, D. Walgraef, Opt. Lett. 23, 1167 (1998).

[22] A. Gatti, L. A. Lugiato, G.-L. Oppo, R. Martin, P. di Trapani, A. Berzanskis, Optics Express 1, 21-30 (1997).

[23] S. Ducci, N. Treps, A. Maître, and C. Fabre, Phys. Rev. A 64,023803 (2001).

[24] H. Kogelnik, T. Li; Appl. Opt. 5, 1550 (1966).

[25] Technical data from the manufacturer (Cristal Laser S. A.).

[26] Antony E. Siegman, Lasers, University Science Books (1986).

[27] A. Heidmann, R. J. Horowicz, S. Reynaud, E. Giacobino, C. Fabre, and G. Camy, Phys. Rev. Letters 59, 255-257 (1987). 